\title{
Bienestar subjetivo y pobreza: Estudio de caso con mujeres en Santiago de Chile'
}

\author{
Subjective well-being and poverty: considerations on a case study \\ with women in Santiago, Chile \\ Bem estar subjetivo e pobreza: Estudo de caso com mulheres \\ em Santiago de Chile
}

Recibido el 25 de agosto de 2014, aceptado el 12 de noviembre de 2014

\author{
Borja Castro Serrano" \\ Chile \\ Marcela Flotts de los Hoyos ${ }^{\prime \prime \prime}$ \\ Chile \\ Cristian Valenzuela ParadalV \\ Chile \\ Paula Vidal MolinaV
}

Para citar este artículo:

Castro Serrano, Borja, Flotts de

los Hoyos, Marcela, Valenzuela

Parada, Cristian, Vidal Molina,

Paula (2014). Bienestar

subjetivo y pobreza:

Estudio de caso con mujeres

en Santiago de Chile. Ánfora,

21(37), 129-150. Universidad

Autónoma de Manizales. ISSN

0121-6538

\section{Resumen}

Objetivo: caracterizar las percepciones de cambio en el bienestar subjetivo de las mujeres de comunas pobres del sur de Santiago que participaron del proyecto promocional en salud mental 'Mujer, Comunidad

Este artículo surge de la investigación "Mujer, Comunidad y Calidad de Vida", trabajada con municipios vulnerables de Santiago de Chile, realizada entre los años 2011 y 2012. Fue ejecutada por la Escuela de Trabajo Social de la Universidad Andrés Bello y financiada por Mutual de Seguridad, a través de su fondo de Dividendo Social, perteneciente a la Dirección de Asuntos Públicos y RSE. Proyecto P-200MUJERCV.

Doctor y Magíster en Filosofía, Psicólogo. Profesor - Investigador Escuela de Trabajo Social-Santiago, Facultad de Ciencias Sociales, Universidad Andrés Bello, Santiago de Chile. Correo electrónico: francisco. castro@unab.cl

Magíster en Desarrollo Humano, Trabajadora Social. Directora de Carrera Trabajo Social, Facultad de Ciencias Sociales, Universidad Andrés Bello, Santiago de Chile. Correo electrónico: mflotts@unab.cl

Magíster en Psicología clínica y Magíster en Dirección y Liderazgo en gestión Educativa, Psicólogo. Profesor adjunto Escuela de Trabajo Social-Santiago, Facultad de Ciencias Sociales, Universidad Andrés Bello, Santiago de Chile. Correo electrónico: cecevp@gmail.com

Dra. En Servicio Social y Magíster en Antropología Social, Trabajadora Social. Profesora adjunta Escuela de Trabajo Social-Santiago, Facultad de Ciencias Sociales, Universidad Andrés Bello, Santiago de Chile. Correo electrónico: pvidal71@yahoo.com 
y Calidad de Vida'. Se trata de precisar su relación con condiciones sociodemográficas, variables metodológicas y habilidades psicosociales. Metodología: estudio de caso, mediante un análisis de datos bivariado (chi cuadrado y correlación) y multivariado (regresión lineal múltiple), se responde qué dimensiones inciden en los niveles de satisfacción vital (variables sociodemográficas; metodológicas; y psicosociales) y si explican o no las percepciones de cambio de bienestar subjetivo de estas mujeres. Resultados: se evidenció que las variables sociodemográficas y metodológicas no inciden ni explican la percepción de cambio en el bienestar subjetivo, no así las variables asociadas al cambio en las habilidades psicosociales, que en un gran porcentaje y con una alta correlación, determinan aquella percepción de cambio. Conclusiones: las variables asociadas a las habilidades psicosociales estudiadas son las que tienen una mayor relación con el bienestar subjetivo, por sobre las sociodemográficas y metodológicas. Y de forma coherente con lo anterior, son el grupo de variables que tienen una mayor incidencia sobre este constructo. Por tanto, el desarrollo de las dimensiones subjetivas en la calidad de vida a través de la promoción de habilidades psicosociales desde un enfoque promocional de salud mental incida en las percepciones del propio bienestar.

Palabras claves: Bienestar social, psicología de comunidades, política social, pobreza, psicología.

\section{Abstract}

Objective: to characterize the perceptions change in the subjective well-being of women who live in poor communities in the south of Santiago and who participated in the promotional project on mental health 'Women, Community and Quality of Life'. It dealt with establishing the incidence of the relation among socio-demographic conditions, methodological variables and psychosocial skills. Methodology: this case study exhibited the dimensions that affect the levels of life satisfaction (demographic, methodological, and psychosocial variables,) by using bivariate (chi square and correlation) and multivariate analyses (multiple linear regression). It also showed whether such dimensions account for the perceptions change related to subjective well-being of these women. Results: neither socio-demographic nor methodological variables affect or account for the perception change of subjective well-being, butvariables associated with change in psychosocial skills, which determine such change to a great extent and in high correlation do. Conclusions: variables associated to psychosocial skills have a greater relation with subjective wellbeing in contrast to socio-demographic and methodological ones. Thus, psychosocial variables have a greater impact on this construct. Therefore, the development of the subjective dimensions in the quality of life by motivating psychosocial skills from a mental health promotion approach influences perceptions of one's well-being.

Keywords: Social well-being, Community Psychology, social policy, poverty, psychology 


\section{Resumo}

Objetivo: caracterizar as percepções de cambio no bem estar subjetivo das mulheres de comunas pobres do sul de Santiago que participaram o projeto promocional em saúde mental 'Mulher, Comunidade e Qualidade de Vida'. Trata se de estabelecer a incidência da relação das condições sócias- demográficas variáveis metodológicas e habilidades psicossociais. Metodologia: estudo de caso, mediante uma análise de dados bivariada (chi quadrado e correlação) e multivariada (regressão lineal múltiplo), se responde que dimensões incidem nos níveis de satisfação vital (variáveis sócio-demográficas; metodológicas; e psicossociais) e si explicam ou não as percepções de cambio de bem estar subjetivo de estas mulheres. Resultados: evidenciou se que as variáveis sóciodemográficas e metodológicas não incidem nem explicam a percepção de cambio no bem estar subjetivo, não assim as variáveis associadas ao cambio nas habilidades psicossociais, que em num grande porcentagem e com uma alta correlação, determinam aquela percepção de cambio. Conclusões: as variáveis associadas às habilidades psicossociais estudadas são as que têm uma maior relação com o bem estar subjetivo, por sobre as sociodemográficas e metodológicas. E de forma coerente com o anterior, é o grupo de variáveis que tem uma maior incidência sobre este constructo. Portanto, o desenvolvimento das dimensiones subjetivas na qualidade de vida a través da promoção de habilidades psicossociais desde um enfoque promocional de saúde mental incida nas percepções do próprio bem estar.

Palabras claves: Bem estar social, psicología de comunidades, política social, pobreza, psicología. 


\section{Introducción}

En Chile, hasta 1973, el Estado asumió la responsabilidad de implementación de las políticas sociales, no sólo en lo que al costo económico significaban. Ese año, con el golpe militar, la estructura de modelo de desarrollo cambió radicalmente (French-Davis y Stallings, 2001). Entre otras consecuencias, la implementación abrupta de un modelo de desarrollo neoliberal, situó al Estado en un rol subsidiario. Los individuos pasaron a ser responsables de su propio bienestar, y por lo tanto, las políticas sociales comenzaron a operar residualmente, destinadas exclusivamente a las personas en situación de extrema pobreza (French-Davis y Stallings, 2001), focalizadas mediante la aplicación de una ficha socioeconómica.

Con el término de la dictadura, en 1990, las políticas sociales impulsadas por el nuevo gobierno ampliaron los grupos vulnerables sobre los cuales el Estado actuaba e incorporaron el componente de participación social (Raczynski y Serrano, 2005). El Estado generó alianzas con el tercer sector y la sociedad civil para fortalecer acciones de transformación.

A partir del año 2000, comenzó a implementarse un nuevo tipo de políticas sociales, denominadas de protección social. Éstas se orientaron a atender la vulnerabilidad socioeconómica y psicosocial de los sectores pobres e indigentes. La lógica detrás de estas nuevas políticas es la de derechos garantizados por ley.

A pesar de que la intencionalidad principal continúa centrada en las condiciones objetivas asociadas a la calidad de vida, especialmente en función del ingreso familiar (Oneto, 2000); en los últimos años, tanto en Chile como en América Latina y el Caribe (Organización Panamericana de la Salud. OPS, 2009), las políticas sociales han intentado incipientemente incorporar la noción de bienestar subjetivo a la calidad de vida apuntando a trabajos promocionales. Un ámbito de intervención pertinente tiene que ver con potenciar la salud mental en mujeres pobres. Ya en los años 90, un estudio de la OPS (Gómez, 1994) hizo referencia a la posición de desventaja de las mujeres respecto a los recursos necesarios para la protección de la salud. Al mismo tiempo, ya se pesquisa si son ellas quienes pueden cumplir un rol protagónico en el mejoramiento tanto de su propia salud como de sus familias y comunidad.

Quince años después, la misma OPS (2009) recomienda en pos de la mejora de la salud mental de la mujer, promover su empoderamiento y participación en la sociedad civil, sobre todo en las más vulnerables económicamente. En el caso chileno, el tercer sector y la sociedad civil han abierto espacios para intervenciones sociales diferenciadas de la oferta estatal, financiadas y ejecutadas por el sector privado con y sin fines de lucro. En ocasiones también, en cooperación y en base a una planificación conjunta con el Estado.

Una de las intervenciones sociales surgidas al alero de la alianza entre lo 
público y el tercer sector en el área de la salud mental local con estrategias de participación social es la de la Fundación El Albergue. Su acción se basó en un método en promoción de salud mental con mujeres de sectores pobres validado por un proceso de investigación que permitió replicarlo durante los años 20092010, financiado con fondos de la Presidencia de la República.

Este programa denominado 'Método Albergue' (Falcone et al., 2009) vincula calidad de vida y bienestar subjetivo con el propósito de promover, a través de talleres grupales vivenciales, habilidades psicosociales cognitivas y emocionales fortaleciendo a mujeres de escasos recursos (ingreso familiar mensual promedio menor a US\$444). El Método Albergue se fundó bajo la idea de cuestionar la lógica del modelo médico tradicional pretendiendo colaborar con él si las respuestas se buscan al interior de quienes se sienten abatidos con lo cotidiano. Así, el modelo buscó una mejoría a partir del contacto, el apoyo y el aprendizaje con los pares, permitiendo que las mujeres lograran no sentirse "pacientes" y los voluntarios que guían el proceso participaran activamente del grupo, sintiéndose facilitador y no un administrador del bienestar. Por tanto, a nivel del enfoque y el tipo de intervención del Método podemos decir que:

(...) el tipo de intervención va en la línea de llevar a la mujer desde su rol pasivo de sufriente, a actriz principal o protagonista en el logro de su bienestar (...) No se trabaja con categorías clínicas o patológicas, sino con categorías preclínicas (...) La metodología usada va en la línea de potenciar el trabajo grupal, facilitar procesos introspectivos, abrir oportunidades de reconocimiento de las propias habilidades y crear un clima de confianza y confidencialidad que permita la participación activa (Falcone et al., 2009, p. 26).

El Método Albergue seleccionó cinco habilidades psicosociales básica por ser deficitarias en mujeres con ánimo depresivo y que se visualizan necesarias para el mejor desarrollo de las vidas femeninas, según criterio clínico de experto. Estas son: autoestima, emocionalidad, comunicación efectiva, creatividad y vínculos colectivos. Por lo tanto, el método, finalmente, se concretiza en el 'Programa de Fortalecimiento para una Vida Saludable' que consta de un total de 10 sesiones grupales, de tres horas aproximadamente cada una, estando compuestas por dos o tres actividades. Cada una de las actividades la realiza el grupo y dos facilitadores, en una lógica que va desde el trabajo del mundo interno hacia el contexto social. Establecemos que las dos primeras sesiones se desarrolla la habilidad de la autoestima; las sesiones tres y cuatro están destinadas a la temática de la emocionalidad; la cinco y la seis ejercitan la comunicación y la asertividad; las sesiones siete y ocho se enfocan en el fomento de la creatividad; finalizando con la sesión nueve y diez que pretende desarrollar los vínculos colectivos a partir del trabajo grupal colaborativo (Falcone et al., 2009).

Este método fue evaluado y sistematizado durante dos años y medio (2006- 
2008) por un equipo especialista en promoción de salud mental, mostrando resultados de un impacto significativo y permanente en el tiempo ${ }^{1}$. Las mujeres percibieron un aumento en su calidad de vida, aumentando su percepción de bienestar subjetivo, disminuyendo los síntomas depresivos (Araya, Rojas, Fritsch, Acuña y Lewis, 2001), mejorando su salud física y mental (Bilbao, Techio y Páez, 2007) como también sus relaciones sociales (Falcone et al., 2009; Bilbao et al., 2007). De este modo y dado los antecedentes, el Método Albergue se implementó nuevamente en el año 2012 gracias a la alianza entre la Escuela de Trabajo Social de la Universidad Andrés Bello (institución privada de educación superior) y Mutual de Seguridad (mutualidad de empleadores sin fines de lucro) creando el proyecto 'Mujer Comunidad y Calidad de Vida”, proceso que permitió el desarrollo del estudio que se presenta en este artículo.

Conceptualmente es pertinente visualizar algunas perspectivas teóricas que despuntan un lugar importante para comprender dónde es que el proyecto se inserta teóricamente. En Chile, así como en otros países de la región, las cifras dan cuentan de la concentración del ingreso, la creciente desigualdad y la desventaja económica de sectores de la sociedad producto del modelo de desarrollo impulsado: el neoliberalismo. Esta situación ha traído consecuencias en el ámbito de la salud física y mental, en la educación y en los problemas sociales propios de una gran población mundial, impulsando una crítica generalizada hacia el modelo imperante (Callinicos, 2003). "Cuanto mayor es la distancia entre la minoría acomodada y la masa empobrecida, más se agravan los problemas sociales, lo que parece ser cierto tanto para los países ricos como los pobres" (Judt, 2011, p. 33).

Chile es un caso particular en este contexto, dado que sus cifras macroeconómicas señalan un crecimiento sostenido en la última década. Sin embargo, este no ha sido del todo inocuo, pues si bien ha significado un incremento en el consumo de la población, expresado a nivel de la alimentación y bienes materiales que son considerados indicadores de bienestar. Esto también ha acarreado consecuencias negativas para la salud de los chilenos. Un ejemplo de ello es el aumento de problemas físicos -obesidad-, psíquicos -estrés y depresión- y culturales sedentarismo y tabaquismo- (Albala, Olivares, Salinas y Vio, 2004).

Dicho lo anterior, es relevante situar, una primera dimensión teórico-filosófica desde los aportes de Axel Honneth. Estos han sido fundamentales a la hora de comprender la relación individual-colectiva y el lugar que ocupa el reconocimiento al interior de una sociedad justa, siendo estos elementos algunos de los pilares relevantes del proyecto y la intervención desarrollada en este estudio.

1. Se precisa que luego de evaluar el Método Albergue, se construyó un programa abreviado de 6 sesiones, incluyendo las mismas habilidades psicosociales y con el mismo enfoque y tipo de intervención. 
Honneth (1997) retomando a Hegel, apunta la existencia de tres esferas de reconocimiento (amor, derecho y social). El daño en alguna de estas esferas, quebranta alguna forma de autorrealización del individuo consigo mismo: la autoconfianza, el auto-respeto y la autoestima. Se establece una vinculación entre la lucha por el reconocimiento y la psicología social, pero también aparece la necesidad de confirmar la existencia institucional de un orden diferenciado de reconocimiento. Lo anterior no es de menor importancia, pues la constitución de la persona es parte de la constitución social de la conciencia individual. Es decir, la formación de la identidad singular queda enlazada con una serie de expectativas normativas que se constituyen a través de las relaciones de reciprocidad social.

(...) el proceso de socialización en general se cumple en la forma de una interiorización de las normas de acción que resultan de la generalización de las expectativas de comportamiento de todos los miembros de la sociedad" y que a través de ello, el individuo 'conquista la capacidad de poder participar en las interacciones normativamente reguladas de su entorno' (Honneth, 1997, p. 98).

Así, tanto la experiencia del amor es clave para la formación de la autoconfianza individual y necesaria para la participación autónoma en la vida pública, como también el entendimiento recíproco de los individuos. Éste, en tanto personas de derecho, se logra mediante un saber moral referido a un "otro generalizado" o a la conciencia de ciertas obligaciones normativas en la comunidad social.

Saliendo de la esfera filosófica, pero no olvidando el tema de la autoconfianza, la reciprocidad de los individuos y su inserción en lo público, la disciplina psicológica también puede ayudar a situar contornos a este trabajo. Dado el estudio realizado el concepto de bienestar subjetivo tiene algo que aportarnos y éste ha tenido una aparición tardía. Desde un inicio ha estado principalmente vinculado al estudio de las condiciones externas que favorecen el bienestar objetivo, lo que en las corrientes anglosajonas se ha denominado el welfare. Diferentes corrientes de pensamiento han asociado lo anterior con las condiciones materiales de los sujetos, lo que se ha conceptualizado como el 'nivel de vida' de éstos (García, 2002).

La introducción del término 'calidad de vida' responde inicialmente a una concepción cuantitativa, y como tal, la orientación de las políticas que intentaron hacerse cargo de este problema apuntó a la modificación de las condiciones socioeconómicas como una forma de mejorar el bienestar de la población (Fadda, Jirón y Bilbao, 2000). Sin desconocer lo crucial de lo antes mencionado, las políticas e intervenciones orientadas de forma exclusiva en esta línea desconocen un elemento central en la calidad de vida: el componente subjetivo.

El desconocimiento de la subjetividad como componente crucial del bienestar puede enmarcarse en la identificación que se ha hecho entre bienestar social -basado en indicadores objetivos como ingresos económicos, acceso a salud, vivienda o condiciones materiales del entorno- y el bienestar económico - 
basado en criterios macrosociales de calidad de vida- (García, 2002). Por lo tanto, se puede decir que la calidad de vida no se agota en la obtención de condiciones materiales mínimas. Si bien existe una necesidad imperiosa de alcanzar condiciones socioeconómicas mínimas para la dignidad de la vida de las personas, no es suficiente para que ellas alcancen el bienestar.

Chile no es la excepción respecto de enfoques 'objetivistas' de intervención en calidad de vida. A la luz de la historia de las políticas sociales chilenas, éstas se han centrado en un intento de apoyar, subsidiar o permitir el acceso a condiciones materiales que, por diversas razones, los sujetos de intervención (personas naturales o familias) no han podido acceder. Pero en la práctica, la percepción subjetiva de la calidad de vida de los beneficiarios de las políticas y de las intervenciones queda sin ser considerada.

En esta misma línea, pero vinculando el bienestar subjetivo desde la perspectiva del feminismo (otro lugar importante para el estudio en cuestión), la preocupación por lo global también ha generado una deuda respecto a la preocupación por lo particular en relación a las mujeres. Como señala Lagarde (2001), se ha promovido una mentalidad idealista que presupone que el mejoramiento de las condiciones colectivas mejora automáticamente a cada quien individualmente.

De esta forma, en oposición a esta omisión de la persona, se plantea un viraje novedoso en el feminismo donde comienza una preocupación por la dimensión personal de la realización trascendente. Desde el feminismo se genera una inversión de la proclama de la década de 1960 y 1970 donde lo personal es político a lo político es personal (Lagarde, 2001). Es desde este cambio que surge la preocupación feminista por la autoestima como una forma de autoconciencia de la propia individualidad y donde también los cambios globales deben considerar la mejora en el bienestar de las mujeres que participan de dicho proceso de cambio. Algunos estudios muestran que el tema de bienestar subjetivo -también entendido como satisfacción vital- implica entenderlo desde roles de género diferentes, cuestión que se hace relevante establecer dado el foco de nuestro estudio (Cárdenas, Barrientos, Bilbao, Páez, Gómez y Asún, 2012).

Habiendo situado elementos que establecen algunos elementos a nivel de lo filosófico, lo psicológico y una nueva mirada a lo feminista, se insiste en las últimas décadas, a nivel global, se ha instaurado un fuerte auge en el reconocimiento del valor de los indicadores subjetivos en la calidad de vida. Se está incorporando en la discusión las nociones de satisfacción de los individuos y los valores personales (Gómez-Vela y Sabeh, 2000), lo que nutre conceptualmente los marcos de la intervención investigada. Este trabajo es preciso en sostener, entonces, que aquí al querer desentrañar temas sobre calidad de vida subjetiva el concepto de bienestar subjetivo toma relevancia. Se entiende este como equivalente al de satisfacción de vida y felicidad, pues todos ellos refieren a una emocionalidad que apuntan a evaluar la calidad de la vida personal: "cuánto nos gusta nuestra propia 
vida” (Bilbao et al., 2007). Esta definición nos permite cimentar un terreno desde dónde situar conceptualmente la intervención realizada.

Así, retomando estos análisis a la realidad chilena, vemos que este tópico también ha puesto el foco de interés en el debate chileno y prueba de ello es el informe sobre Desarrollo Humano centrado en el bienestar subjetivo del año 2012 del Programa de Naciones Unidad para el Desarrollo (PNUD), el cual plantea que:

(...) tanto la dimensión objetiva como la subjetiva del bienestar son importantes. Y que una de las finalidades de los indicadores de bienestar debe ser la evaluación que hacen las personas de sus vidas, de sus experiencias y sus prioridades (...) lo que es un aspecto central para el desarrollo (PNUD Chile, 2012, p. 42).

Entonces, si se ha de considerar el problema de la calidad de vida ineludiblemente anudado al bienestar subjetivo, resulta necesario establecer un marco de acuerdo sobre esta noción. El informe del PNUD trabaja desde una perspectiva, que si bien no agota la complejidad del problema de la subjetividad, ayuda a avanzar en una definición, pues pretende considerar el bienestar subjetivo como una meta a alcanzar dentro del desarrollo. Por esto, se debe comenzar por un concepto amplio de la subjetividad que incluya tanto la dimensión personal como la referida a la sociedad. De esta manera, se puede asumir que la subjetividad está configurada desde el propio juicio que las personas hacen sobre sí misma como también por el que tiene de la sociedad en que viven. Y ambos son relevantes y pueden no coincidir, de ahí la importancia de no atender a una sola sino a todas las dimensiones de la subjetividad (PNUD Chile, 2012).

En coherencia con la posición del PNUD, en esta articulación entre lo personal y lo societal, podemos agregar otros matices describiendo el modelo de Lawton (García, 2002). Éste trabaja la calidad de vida centrada en elementos psicológicos o subjetivos, pero influenciados por el ambiente social. Se proponen cuatro grandes áreas articuladas: medio-ambiente objetivo, percepción de la calidad de vida, bienestar psicológico y competencias conductuales. El medioambiente objetivo, que en rigor sería el único elemento completamente externo o no subjetivo del modelo, hace referencia a las características físicas del entorno de la vida de los sujetos. La percepción de calidad de vida incluye el concepto de la satisfacción de la persona consigo misma y con la familia, amigos, trabajo, actividades y lugar donde vive. El bienestar psicológico estaría ligado, por un lado, a la relación entre los objetivos deseados y los alcanzados, y por otro, al ánimo y los estados afectivos. Por último, aparece el componente de las competencias conductuales, las cuales consistirían en una capacidad funcional y estado de salud adecuado, un buen comportamiento social y funciones mentales preservadas. 
Aquí resulta clave insertar la noción de determinantes psicosociales, lo que permite articular el modelo de calidad de vida con las nociones de bienestar subjetivo y de salud.

(...) los determinantes psicosociales de la salud son un conjunto amplio y heterogéneo de factores asociados al proceso de salud - enfermedad y la calidad de vida de las personas (...) se puede diferenciar entre aquellos internos o propios del individuo vinculados al comportamiento y los externos, referidos a las relaciones interpersonales y sociales, así como la cultura y las condiciones de vida (Ministerio de Salud (MINSAL), 2002, p. 108).

Así, para la mejora de los Estados de salud muchas veces el desarrollo de recursos psicosociales es tan relevante que trasciende el campo biológico. Y si bien bienestar subjetivo no es igual a salud mental, sí se puede decir que a mayor bienestar subjetivo las personas se sienten más productivas, creativas y sociables, mejorando los determinantes de la salud; potencia la felicidad, generando capacidad de amar, trabajar, relacionarse con otros, sintiéndose a gusto en su medio social. Potenciar el bienestar subjetivo ha demostrado que ayuda a bajar los indicadores de estrés mediante reforzamiento de los estados emocionales (García-Viniegras y González, 2000) como también los síntomas depresivos y angustiosos (Falcone et al., 2009). El último reporte sobre felicidad de la Organización de las Naciones Unidas (ONU) (Helliwell, Layard y Sachs, 2012), establece que la salud mental es el factor más potente unitariamente que afecta la felicidad y el bienestar de las personas en cualquier tipo de país.

Si se profundiza la relación entre bienestar subjetivo y la potenciación de factores psicosociales, se debe retomar la Primera Conferencia Internacional de Promoción de Salud en la Carta de Ottawa (2001). Aquí se define que la metódica de la promoción es definida como la capacidad de las personas para que puedan proporcionarse los medios en pos de una mejora de salud y así lograr mayor grado de autonomía. Luego, desde la 5 ta Conferencia Internacional sobre Promoción de la Salud (OMS, 2000) se declara la necesidad de modificar las intervenciones en salud desde un enfoque promocional. Los lineamientos de la OMS pretenden conceptualmente situar al individuo en el centro de su mejoría, en la lógica de prevenir la enfermedad potenciando sus propios recursos hacia una mejor calidad de vida, incluyendo las categorías subjetivas pues son tan relevantes para el bienestar individual y social (Schwartzmann, 2003).

Esta situación se complejiza ingresando variables como la pobreza, el género, la desigualdad medida por el coeficiente de Gini (Organización para la Cooperación y el Desarrollo Económica. OCDE, 2011 ); ahí es posible percatarse que las inequidades estructurales en Chile también se encuentran en el ámbito de la calidad de vida y el bienestar subjetivo. Si se toma la pobreza y el género como factores relevantes, algunos estudios muestran que al hacer una diferenciación 
entre la prevalencia de los síntomas comunes y los síntomas psiquiátricos, ambos tienen mayor prevalencia en la población femenina. Las preocupaciones, la fatiga y la irritabilidad son los síntomas comunes con mayor prevalencia en mujeres. Entre los síntomas psiquiátricos, los más comunes entre las mujeres son los depresivos, ansiosos y fóbicos (Araya et al., 2001). Y por otro lado, encontramos que la pobreza es un factor determinante en la salud, y no solo en Chile sino, en general, en América Latina y el Caribe (Legetic, 2004).

La preocupación por la salud, el bienestar y la equidad de género asociada a la pobreza, es una vinculación ya trabajada desde otras organizaciones. De acuerdo con la OPS (2009) en el marco del Plan de Acción de Adolescentes y Jóvenes 2010-2014, resulta una prioridad para Latinoamérica y el Caribe el empoderamiento como agente clave para el cumplimiento de los Objetivos del Milenio. Se identifican la equidad de género y el empoderamiento de las mujeres como una condición para reducir la pobreza y mejorar la salud y el bienestar de las personas. Es decir, la potenciación del individuo en el sentido de ganar poder o autonomía respecto de su propia vida. En este sentido, se plantea que el generar acciones que apunten al empoderamiento de las mujeres tiene un efecto directo sobre la salud y bienestar de ellas, cuestión que se transforma en un pivote para ayudar al desarrollo de la región. Este enfoque implica reconocer que la potenciación de las capacidades individuales, además de beneficiar subjetivamente a las mujeres, es también una herramienta estratégica para el desarrollo de la comunidad.

Dado lo anterior y con base en lo expuesto en los inicios de este apartado, el presente estudio se basó en la experiencia del Método Albergue en tanto su metodología abre oportunidades de reconocimiento de las propias habilidades, cuestión que pretende potenciar el bienestar subjetivo en una población femenina y en situación de pobreza. Así, el objetivo general del estudio es caracterizar las percepciones de cambio en el bienestar subjetivo de las mujeres participantes del Método Albergue, precisando su relación con condiciones sociodemográficas, variables metodológicas y habilidades psicosociales.

\section{Metodología}

La pregunta que guía la presente investigación consiste en conocer cuáles son las dimensiones que inciden en el cambio de la percepción de bienestar de las mujeres que participaron de los talleres Método Albergue durante el año 2012. Particularmente, en virtud que el método ha mostrado funcionar respecto a mejorar la percepción de bienestar de las mujeres, se busca responder si las variables sociodemográficas (edad, nivel educacional, ocupación, hijos y estado civil), las metodológicas (centro de derivación, cantidad de sesiones -6 o 10 
sesiones-) y las habilidades psicosociales trabajadas (autoestima, emocionalidad, comunicación efectiva, creatividad y vínculos colectivos) inciden y explican o no las percepciones de cambio de bienestar subjetivo (en términos de desarrollo de las capacidades internas).

Como hipótesis se plantea que la subjetividad de las mujeres respecto a su bienestar es posible de ser intervenida y una intervención con las características del Método Albergue tiene efectos positivos en las personas a nivel de su calidad de vida.

Respecto al diseño metodológico, el presente estudio corresponde a un análisis de casos que considera la percepción de las 185 mujeres que participaron de los talleres ejecutados mediante el Método Albergue en las comunas de El Bosque, Pedro Aguirre Cerda y La Granja, de Santiago de Chile. Cabe señalar que no se trata de una muestra probabilística que pretenda representar a las mujeres chilenas que viven en situación de pobreza. La finalidad es poder analizar las percepciones de las participantes del caso en que este estudio se centra, considerándolas como una tendencia aplicable a contextos de condiciones semejantes. Las características generales de las mujeres que participaron son las siguientes: un 80 por ciento recibe un ingreso familiar menor a 600 dólares mensuales; la mayoría tiene entre 35 y 55 años, y más del 60 por ciento ha cumplido su enseñanza secundaria. El 47 por ciento vive con su pareja y el 73 por ciento es dueña de casa. Por último, sólo el 7,9 por ciento no tiene hijos.

Para resolver la pregunta se utilizaron los resultados de la encuesta aplicada a cada mujer ex - antes y ex - post de los talleres, lo que permitió conocer la percepción que las mujeres señalan respecto a su bienestar subjetivo, en los dos momentos de medición. Por lo tanto, a pesar que la complejidad del fenómeno abordado implica sin duda la acción de múltiples dimensiones. Lo que recoge centralmente este estudio es el cambio que las mismas mujeres perciben respecto a su bienestar antes y después de su participación en el programa. Concretamente, la evaluación de perfil y la medición de impacto individual se realizaron a través del instrumento del Método Albergue, el cual fue construido el año 2006.

Este es un cuestionario semi-estructurado (encuesta social) y consta de 46 preguntas precisas con alternativas de respuestas, pero también de preguntas abiertas que permiten flexibilizar las opciones de respuestas desde las opiniones vertidas por las encuestadas. Así, indaga y evalúa la percepción y valoración de las cinco variables investigadas, además de examinar el bienestar percibido por las examinadas. Los valores de consistencia interna, mediante la aplicación del alfa de Cronbach, son altos, así 0,89 para la etapa Ex- ante, 0,91 para la etapa de Resultados y 0,93 para la etapa Ex- post. Además, en aquel momento se certificó la validez del instrumento realizando un testeo del cuestionario a un grupo de personas con características similares a la muestra seleccionada, con la finalidad de comprobar la comprensión de las preguntas por parte del entrevistado, 
ubicar preguntas que presentan rechazo, eliminar o reemplazar preguntas que induzcan respuestas en una cierta dirección, no emplear términos técnicos que las personas entrevistadas puedan desconocer, establecer tiempo de duración de la encuesta y conocer la cantidad óptima de preguntas que acepta el beneficiario (Falcone et al., 2009).

De esta forma, el análisis de datos que se llevó a cabo utilizó la información contenida en la base de datos que sistematizó las encuestas aplicadas. Las técnicas de análisis de la información utilizadas fueron el análisis bivariado (chi cuadrado de Pearson, correlación de Spearman y V de Cramer) y el análisis multivariado (regresión lineal múltiple).

\section{Resultados y discusión}

Se evidenció que además de las mediciones objetivas y/o absolutas de la calidad de vida se requieren fortalecer aquellos espacios de intervención pública y privada orientadas a promover diversas habilidades psicosociales (mediante intervenciones como las del Método Albergue) que provocan cambios en cómo las personas, en este caso mujeres, perciben su propio bienestar. A continuación, se presenta en primer lugar los datos descriptivos arrojados a nivel estadístico. Luego, para aportar a la discusión e intentar superar los análisis de los resultados numéricos, se mostrarán reflexiones que nutren el concepto de bienestar subjetivo en un horizonte de pobreza visualizando su impacto cuando se trabajan habilidades psicosociales. Se finaliza con algunos análisis que proponen un desafío a nivel de la política social y la institucionalidad cuando se trabaja con intervenciones que son promotoras del bienestar subjetivo.

En relación con los datos estadísticos, el análisis bivariado arrojó que existe una relación estadísticamente significativa entre las variables percepción sobre habilidades psicosociales (autoestima, emocionalidad, comunicación, creatividad y vínculos con el entorno) y la percepción de cambio en el bienestar subjetivo de las mujeres. Además, se puede establecer que en cada una de las habilidades la intensidad de la relación es alta.

Este análisis precisa, cuantitativamente, la importancia del primer párrafo descrito en estos resultados respecto al bienestar psicosocial y la posibilidad generar intervenciones desde este prisma. Además, lo connota con mayor fuerza cuando se evidencia que no existe una relación estadísticamente significativa tanto entre las variables sociodemográficas (edad, nivel educacional, ocupación, hijos y estado civil) y la percepción de cambio en el bienestar subjetivo; como también entre las variables metodológicas (derivación y número de sesiones) y la percepción de cambio en el bienestar subjetivo. Esto último implica que tanto el tipo de derivación de las mujeres (sector salud o sede social) como el 
número de sesiones trabajadas por ellas (taller de diez o seis sesiones) no incide en la percepción de cambio que ellas tienen respecto al cambio en su bienestar subjetivo.

Por otro lado, a nivel de los datos arrojados en el análisis multivariado (tabla uno), se puede señalar que dentro de las variables sociodemográficas (modelo uno), solo el nivel educacional y el estado civil tienen un impacto estadísticamente significativo en la percepción de cambio de bienestar subjetivo. No obstante, en su conjunto, las variables sólo explican el 1,8 por ciento de aquella percepción.

A nivel de las variables metodológicas (modelo dos), sólo el número de sesiones tiene impacto estadísticamente significativo en la percepción de cambio de bienestar subjetivo. Pero, en su conjunto, explican apenas en 0.9 por ciento de la percepción de cambio.

Y aquí la constatación más importante y que va en la línea del análisis bivariado: se observó que todas las variables psicosociales tienen un impacto sobre la percepción de cambio en el bienestar subjetivo de las mujeres. La integración de estas variables al análisis, hace aumentar a un 60.2 por ciento (modelo tres) el $\mathrm{R}$ cuadrado corregido, lo que habla de un fuerte impacto. Así, aquellas mujeres que aumentan su percepción de cambio en cada una de las habilidades psicosociales perciben también un aumento en su bienestar subjetivo. Esto muestra la clara sintonía con otros estudios que estipulan que el mejorar a nivel de bienestar subjetivo a nivel individual supone una mejora en la autoestima, baja en la afectividad negativa aumentándola como también claras mejoras en la autoestima (Bilbao et al., 2007).

Lo anterior permite pensar que el bienestar no sólo atraviesa condiciones estándar o absolutas respecto de las condiciones necesarias o mínimas para vivir dignamente, sino que requiere, necesariamente, un trabajo que considere las subjetividades tanto individuales como de la persona en relación con su entorno. La OMS $(2001,2000)$ y otros organismos internacionales (OPS, 2009), ya han planteado la importancia de enfocar las intervenciones en los propios recursos de las personas hacia una mejor calidad de vida, incluyendo ya no sólo dimensiones objetivas (variables socioeconómicas), sino que también categorías subjetivas que son relevantes para el bienestar individual y social (Schwartzmann, 2003).

A sí mismo, la tabla uno muestra, también, que dentro de las habilidades psicosociales que inciden principalmente en la percepción de cambio en el bienestar subjetivo son las variables de creatividad y vínculos sociales. La creatividad, como una habilidad que puede convertirse en un gran recurso emocional para buscar soluciones y resignificar las condiciones de vida; y los vínculos sociales como una habilidad que permite relacionarse con otros, fortalecer las redes, mejorar el capital social y buscar en el entorno cercano lo que no ofrecen las políticas sociales (Bilbao et al., 2007). 
En un análisis más profundo, a nivel de la percepción de bienestar subjetivo, la relevancia de las habilidades psicosociales puede ser un tema importante para abordar la pobreza en un horizonte del desarrollo. Por tanto, teniendo en perspectiva los datos estadísticos, se quiere enfatizar que este estudio no pretende denostar los esfuerzos del Estado y de otros organismos del tercer sector que tienen por objetivo nivelar las condiciones objetivas y materiales de aquella población más desventajada de Chile, sino más bien refuerza la idea de que no es suficiente poner los esfuerzos exclusivamente en esa dimensión de la ayuda entregada a aquellos grupos más vulnerables.

Actualmente, se hace necesario ir hacia un 'más allá de las condiciones materiales, pero sin dejarlas de lado. Es decir, las intervenciones que pretenden mejorar la calidad de vida de las personas más desventajas de Chile deben incorporar el manejo y el trabajo de las habilidades psicosociales. Su concientización y potenciación enriquece las posibilidades de esas personas en su propio mundo, lo que tendería a explicar el cambio en la percepción del bienestar subjetivo (mejora de autoestima y emocionalidad) como también su mejora en ciertas dimensiones de la salud mental (mejora sintomatológica y en sus relaciones interpersonales) (MINSAL, 2002). “(...) la promoción de la salud (...) permite desarrollar y potenciar los recursos saludables que toda persona posee, logrando una mejora significativa en su calidad de vida" (Falcone et al., 2009, p. 33).

Según la vivencia registrada del universo de mujeres que participaron del estudio, se pesquisa que a partir del desarrollo de las habilidades psicosociales -las que mejoran la percepción sobre el bienestar subjetivo- se hace posible movilizar aspectos emocionales y cognitivos que les permiten modificar actitudes y conductas (García, 2002), logrando trazar redes que las insertan comunitariamente. Esto genera, en algunas de ellas, que en un mediano plazo mejoren condiciones materiales (mejores trabajos, aperturas de negocios, etc.) para su propio mundo de vida; se averigua un empoderamiento asociado a la inserción en un nivel intermedio u organizacional (Garretón, 2001).

La percepción de cambio en su bienestar subjetivo, asociado a la movilización de recursos emocionales (mundo interno) y sociales (capital social), les hace abrir alternativas de modo creativo. En este sentido, no es extraño que las habilidades psicosociales estudiadas que más explican el cambio en la percepción del bienestar subjetivo resalten los vínculos sociales como la creatividad. Esta última les permite a las mujeres potenciar la motivación, la capacidad transformadora y la iniciativa personal para modificar las problemáticas cotidianas a las que se ven expuestas (Falcone et al., 2009).

Dicho de otro modo, el desarrollo de estas habilidades psicosociales (y de sobre manera la creatividad como los vínculos), potenciadas en un espacio de acogida que privilegia el vínculo de unas con otras -dado la metodología del 
Método Albergue- son explicativas en un alto porcentaje lo que da para pensar el bienestar subjetivo como motor de cambio. Así, se puede entender “(...) que la pobreza no solamente está centrada en la carencia de bienes físicos y de servicios básicos, se da mucha importancia a las deficiencias de bienes socio-emocionales que es uno de los elementos del paradigma del capital social" (Forni, Siles y Barreiro, 2004, p. 2).

Lo anterior respalda la importancia de tener presente la variable del bienestar subjetivo y los alcances de la propia subjetividad en la posibilidad de generación de vínculos significativos con el medio; como a su vez, en la apertura de posibilidades para la mejora de las condiciones materiales a través de actos creativos y de vínculos seguros, factores que mejoran la calidad de vida de estas mujeres. Así, comprender la potencia de trabajar sobre el bienestar subjetivo indica un paso relevante cuando se quieran pensar intervenciones o programas que impacten en la calidad de vida de los usuarios. Esto obliga a investigar las nociones de calidad de vida articulando tanto sus corrientes objetivas como las subjetivas (Gómez-Vela y Sabeh, 2000; PNUD, 2012), sin desmedro de alguna de ellas.

En esta línea, se puede plantear que los resultados de este estudio ponen en juego algo clave: al trabajar la noción de calidad de vida con énfasis en el bienestar subjetivo se está también instalando un eje para el desarrollo de los países latinoamericanos. En otras palabras, cuando se visualiza que las mujeres entienden que su propio bienestar no sólo depende de sentirse mejor, sino también de avanzar hacia lo que la propia sociedad ofrece para lograr un mayor bienestar personal -relación con la creatividad y los vínculos colectivos como habilidades psicosociales trabajadas- se abre un camino a explorar y repensar las nociones de subjetividad y desarrollo. Es decir, el estudio entreabre un desafío que pretende comprender la inserción del bienestar subjetivo de un modo amplio para que logre ser parte del desarrollo, considerando una cierta subjetividad que incluye un juicio sobre sus capacidades propias/personales como un juicio respecto a la sociedad en que se desenvuelven comprendiendo qué es lo que ésta le puede ofrecer para su propio bienestar (PNUD, 2012).

Finalmente, estos resultados a nivel de bienestar subjetivo y pobreza, impactan la política social y le imponen algunos desafíos tanto a nivel de las instituciones que la piensan y la ejecutan como a las disciplinas que la practican y la teorizan. Por tanto, mirados los resultados en función de las políticas sociales chilenas cobran mayor sentido y significación, especialmente en un contexto neoliberal donde el Estado reduce su ámbito de acción y compromiso de protección con amplios sectores de la sociedad. La política social, por lo tanto, no entrega los recursos simbólicos y materiales para superar la situación de pobreza ni para generar en la población claros sentidos de bienestar subjetivo. Por lo tanto, una 
intervención que aborde y se preocupe metodológicamente de esto, complementa las acciones del Estado en dichos aspectos.

Así, se pueden vislumbrar múltiples desafíos para las políticas sociales. Los que más claramente se puede advertir a partir de los resultados de este estudio de caso, se relacionan con la relevancia de la interinstitucionalidad y la interdisciplinariedad como dimensiones que ayudan a promover la incorporación de la subjetividad para promover el bienestar de las personas. En ese sentido, el énfasis que se ha dado a nivel discursivo sobre la importancia que adquieren las redes institucionales y la intersectorialidad en la intervención social se concreta en experiencias como las que se ha descrito en el presente estudio. Aquí, lo que se ha puesto en juego son la unidad de la diversidad institucional, que poseen lógicas y estructuras organizacionales distintas, pero que han sido capaces de acordar un trabajo conjunto bajo la unidad de una intervención a favor de los sectores desprotegidos socialmente. El éxito de la intervención demuestra que es posible generar alianzas interinstitucionales bajo un objetivo claro, lo cual viene a permitir no solo complementar recursos sino también experticias de cada uno de los sectores (ONU, 2005). El trabajo con los sectores pobres de la sociedad no los resuelve una sola institución. En ese sentido, esta experiencia demuestra lo que la literatura y los discursos presidenciales han mencionado desde hace un tiempo a la fecha.

Por su parte, el campo complejo de la pobreza y las desigualdades puede ser comprendido a mayor cabalidad desde la interdisciplinariedad (ONU, 2005), como a su vez, las propuestas de soluciones para abordarlos. En este caso, siendo la subjetividad y el bienestar humano aspectos que contienen variadas características y dimensiones, la mirada interdisciplinaria e intersectorial facilita la consideración de tal complejidad al momento de intervenir. En tal sentido, las políticas sociales deben dejar de ser construidas verticalmente desde un aparato central. No pueden construirse alejadas de la interlocución con las diversas instituciones que funcionan en un territorio como de los diversos profesionales que intervienen en lo social. No pueden dejar de considerar la voz y necesidades múltiples de los sectores sociales. Por ello, se hace más evidente que la pobreza no sólo es un problema material, de ingresos o de ingeniería social; hay que entender la necesidad de incorporar las habilidades sociales como eje central para pensar mejores condiciones de vida para las personas.

\section{Conclusiones}

Este estudio aporta a constatar que las variables asociadas a las habilidades psicosociales estudiadas son las que tienen una mayor relación con el bienestar subjetivo, por sobre las sociodemográficas y metodológicas. Y de forma coherente 
con lo anterior, son el grupo de variables que tienen una mayor incidencia sobre este constructo.

Por lo tanto, a pesar de que las condiciones materiales se mantuvieron inalteradas, el potenciar las capacidades individuales de las mujeres en un contexto de acogida grupal y con un enfoque promocional, tuvo una incidencia potente en la percepción respecto al cambio en el bienestar subjetivo, posterior a la intervención. Los resultados aquí expuestos reafirman la posición de que el factor subjetivo es crucial para la modificación de la calidad de vida de los individuos (García, 2002).

Así es como también cobra relevancia la idea de trabajar el desarrollo de habilidades psicosociales desde un enfoque promocional de salud mental. A la luz de estos resultados y siguiendo la línea de lo antes planteado, es posible aventurar que las mujeres al ver potenciadas sus habilidades psicosociales, modifican la percepción de bienestar considerando las capacidades que tienen para enfrentar la realidad cotidiana que les toca vivir. E incluso, esto puede ayudarlas a generar cambios concretos en sus condiciones materiales de vida.

Cabe mencionar otros estudios que vinculan bienestar subjetivo, salud mental y ambientes sociales (Cuadra y Florenzano, 2003), donde se muestra que los contextos sociales son clave en este tipo de intervenciones para potenciar el bienestar subjetivo como determinante de la salud mental. Identificando metodologías empíricas, se comprueba que al potenciar lo competente, las relaciones interpersonales y la autonomía son una clave predictiva del bienestar subjetivo y desarrollo social, lo que también se ve reflejado en este estudio. De aquí la importancia de poder vincular estos temas con la política social.

Así, lo auspicioso de los resultados ratifica la necesidad, en un contexto tan desigual en estas materias como lo es el chileno (Castillo, 2012), procurar que las intervenciones que se generen consideren la mirada subjetiva en intervenciones sobre calidad de vida, pudiendo incorporar la noción de bienestar subjetivo de modo paralelo al mejoramiento de las condiciones materiales. Esto permite visualizar la importancia de conjugar aspectos psicológicos, sociales y comunitarios a la hora de pensar intervenciones que pretenden mejorar la calidad de vida de personas en situación de vulnerabilidad social y psíquica.

Con base en lo anterior, se cree que alcanzar el desarrollo social no es cuestión solamente de datos financieros, números azules y macro-indicadores económicos, también es cuestión de sentidos, sensibilidades y subjetividades particulares, que en países como Chile y otros latinoamericanos, se manifiestan muchas más veces de las que quisiéramos en horizontes de precariedad y descuido del bienestar personal y social. 


\section{Agradecimientos}

Los autores agradecen a Dra. María Elisa León, gerenta de Investigación, Innovación y Desarrollo de Mutual de Seguridad como al Dr. Christian Vuskovic, director técnico de la Unidad de Calidad de Vida de Mutual de Seguridad por sus valiosos aportes. Además, se agradece al equipo de investigación: Francisca Fierro, María Luisa Tapia, Romina Neira, Vania Pizarro, Nickolás Alarcón, Daniela Barra, Susana Canales, Marisol Contreras, Carolina Delgado, Valeska Fritz, Paulina Gajardo, Dania Guerrero, Paola Pezoa, Katherine Silva, Angélica Solorza, Gissel Trujillo, Alan Muñoz, Tamara Carrillo, Carolina Garrido, Natalia Valenzuela, Nicole Barra, Pamela Rojas.

\section{Referencias}

Albala, C., Olivares, S., Salinas, J., y Vio, F. (Eds.) (2004). Bases, prioridades y desafios de la promoción de la salud. Santiago de Chile: Universidad de Chile.

Araya, R., Rojas, G., Fritsch, R., Acuña, J. y Lewis, G. (2001). Common mental disorder in Santiago, Chile. British Journal of Psychiatry, 178, 228-233.

Bilbao, M. A., Techio, E. M. y Páez, D. (2007). Bienestar Subjetivo, Cultura y Valores Personales: estado de la cuestión y síntesis meta-analítica de estudios. Revista de Psicología - Edición Especial en Psicología Cultural y Transcultural, 25(2), 233-276.

Callinicos, A. (2003). Igualdad. Madrid: Siglo XXI.

Cárdenas, M., Barrientos, J., Bilbao M.A., Páez, D., Gómez, F. y Asún, D. (2012). Estructura Factorial de la Escala de Satisfacción con la Vida en una muestra de estudiantes universitarios chilenos. Revista Mexicana de Psicología, 29 (2), 157-164.

Castillo, J.C. (2012). Is Inequality Becoming Just? Changes in Public Opinion about Economic Distribution in Chile. Bulletin of Latin American Research, 31(1), 1-18.

Cuadra, H. y Florenzano, R. (2003). El bienestar subjetivo: hacia una psicología positiva. Revista de Psicología, 12(1), 83-96.

Falcone, J., Castro, B., Serrano, P., Ortiz, V., Toral, M., Vuskovic, Ch. \& Gúzman, C. (2009). Promoción de la calidad de vida: Evaluación de impacto del programa 'Fortalecimiento para una vida saludable'. International Journal of Psychological Research, 2(1), 24-34. 
Fadda, G., Jirón, P. y Bilbao, M. A. (2000). Evaluación de la calidad de vida desde la perspectiva bifocal de 'medio ambiente-genero'. El caso de un barrio en Santiago. Boletín del Instituto de la Vivienda, 15(1), 120-131.

Forni, P., Siles, M., y Barreiro, L. (2004). ¿Qué es el Capital Social y cómo Analizarlo en contextos de Exclusión Social y Pobreza? The Julian Samora Research Institute, 35, Michigan: Michigan State University.

French-Davis, R. y Stallings, B. (2001). Reformas, crecimiento y politicas sociales en Chile desde 1973. Santiago de Chile: CEPAL/Ed. Lom.

García, M. (2002). El Bienestar Subjetivo. Revista Escritos de Psicología; 6, 18-39. Recuperado de http://www.escritosdepsicologia.es/descargas/ revistas/num6/escritospsicologia6_analisis 1.pdf

García-Viniegras, C. y González, I. (2000). La categoría del bienestar psicológico, su relación con otras categorías sociales. Revista Cubana Med Gen Integr; 16(6), 586-592.

Garretón, M.A. (2001). Cambios sociales, actores y acciones colectivas en América Latina. Santiago de Chile: Serie Políticas Sociales, CEPAL, Naciones Unidas, División de Desarrollo Social.

Gómez, E. (1994). La salud y las mujeres en América Latina y el Caribe. Viejos problemas y nuevos enfoques. Mar del Plata: Serie Mujer y Desarrollo 17, Organización Panamericana de la Salud (OPS).

Gómez-Vela, M. y Sabeh, E. (2000). Calidad de vida. Evolución del concepto y su influencia en la investigación y práctica. Salamanca: Instituto universitario de integración en la comunidad, INICO.

Helliwell J., Layard R. y Sachs J. (2012). World Happiness report, Nueva York: Earth Institute.Judt, T. (2011). Algo va mal. Madrid: Taurus.

Honneth, A. (1997). La lucha por el reconocimiento. Por una gramática moral de los conflictos sociales. Barcelona: Editorial Crítica.

Lagarde, M. (2001) 'Autoestima y Género' en Cuadernos Inacabados 39, Claves feministas para la autoestima de las mujeres, s/i.

Legetic, B. (2004). La promoción de salud en América Latina y el Caribe. En Albala, C., Olivares, S., Salinas, J. \& Vio, F. (Eds.) Bases, prioridades y desafios de la promoción de la salud. (pp. 8-19). Santiago de Chile: Universidad de Chile.

Ministerio de Salud (2002). Los Objetivos Sanitarios para la Década 20002010. Santiago de Chile: División de Rectoría y Regulación Sanitaria, Departamento de Epidemiología. 
Organización para la Cooperación y el Desarrollo Económica, OCDE (2011). Society at a Glance 2011 - OECD Social Indicators. Recuperado de www. oecd.org/els/social/indicators/SAG,http://statlinks.oecdcode. org/812011041P1G024.XLS

Oneto, L. (2000). Viejos referentes argumentativos en políticas sociales: hacia un modelo de análisis de sus sedimentaciones. Valparaíso: Universidad Católica de Valparaíso.

Organización de las Naciones Unidas. ONU (2005). Objetivos de desarrollo del milenio. Una mirada desde América Latina. Santiago de Chile.

Organización Mundial de la Salud. OMS (2001). Carta Ottawa para la promoción de la salud. Salud PúblicaEduc Salud, 1(1), 19-22.

Organización Mundial de la Salud. OMS (2000). Quinta Conferencia Mundial de Promoción de la Salud; promoción de la salud: hacia una mayor equidad. Recuperado de http://www.cepis.org.pe/bvsdeps/fulltext/conf5.pdf

Organización Panamericana de Salud. OPS (2009). Empoderamiento de Mujeres Adolescentes: un proceso clave para lograr los Objetivos del Desarrollo del Milenio. Recuperado de http://new.paho.org/hq/dmdocuments/2010/ Empoderamiento\%20de\%20Mujeres\%20Adolescentes.pdf

Programa de la Naciones Unidas por el Desarrollo. PNUD Chile (2012). Desarrollo humano en Chile. Bienestar subjetivo: el desafio de repensar el desarrollo. Santiago de Chile.

Raczynski, D. y Serrano, C. (2005). Las políticas y estrategias de desarrollo social: aportes de los años 90 y desafíos futuros. En Meller P. y Landerretche, O. (Eds.). La paradoja aparente. Equidad y eficiencia: resolviendo el dilema (pp. 225-283). Santiago de Chile: Taurus.

Schwartzmann, L. (2003). Calidad de vida relacionada con la salud: Aspectos Conceptuales. Revista Ciencia y Enfermería, 9(2), 9-21. 
Tabla 1. Determinantes de la Percepción de Cambio en el Bienestar Subjetivo

\begin{tabular}{lccc}
\hline Variables & Modelo 1 & Modelo 3 & Modelo 3 \\
\hline Variables sociodemográficas & & & \\
Nivel educacional & 071 & .078 & .034 \\
Ocupación & - & - & .017 \\
Hijos & - & .060 & .003 \\
Estado Civil & .051 & .052 & - \\
Edad & - & - & - \\
\hline Variables metodológicas & & & \\
Número de sesiones & & 188 & .095 \\
Derivación & & - & - \\
\hline Variables percepción habilidadespsicosociales & & & .190 \\
Autoestima & & & .149 \\
Emocionalidad & & & .153 \\
Comunicación & & & .369 \\
Creatividad & & & \\
Vínculos & .028 & .055 & .645 \\
\hline Rcuadrado & .018 & .009 & .602 \\
Rcuadrado corregido & & & \\
\hline
\end{tabular}

Fuente: elaboración propia, en base a encuestas aplicadas por Método Albergue (Diciembre 2011 - Agosto 2012). N=185. Se muestran sólo los valores estadísticamente significativos. 